\title{
Profile: Karachi Health and Demographic Surveillance System of Pakistan (KHDSS)
}

\author{
Komal Naeem*, Muhammad llyas, Urooj Fatima, Momin Kazi, Fyezah Jehan, \\ Yasir Shafiq, Murtaza Taiyab, Usma Mehmood, Rashid Ali, Anita K. Zaidi and \\ Muhammad I. Nisar
}

Pediatrics and Child Health, Aga Khan University, Karachi, Pakistan

\section{Objective}

The mandate of establishing this DSS is to provide a research platform for both observational and interventional studies, with focus on maternal and child health, which could influence decision-making and planning for health strategies at local, national and international levels.

\section{Introduction}

The Karachi Health and Demographic Surveillance System was set up in year 2003 by the Department of Pediatrics and Child Health of the Aga Khan University, Karachi, Pakistan, in four peri-urban low socioeconomic communities of Karachi and covers an area of 17.6 square kilometers.(Figure 1).

\section{Methods}

Total population currently under surveillance is 299,009 for which a record of births, deaths, pregnancies and migration events is maintained by two monthly household visits. At each re-enumeration, Community Health Workers move through the area using GIS-derived maps and collect the information from households and conduct verbal autopsies for stillbirths and deaths of children under the age of five and adult female. Primary Health Care centre at each site provide free care to children under 5 .

\section{Results}

The demographic characteristics for the year 2016 are summarized in Table 1. The main demographic indicators for a period of five years enable us to study the trends of population dynamics and reasons for the change in the rates of stillbirth, under 5 children mortality and maternal mortality (Table 2). Under 5 mortality rates peaked in 2013 and 2016 due to measles epidemic. Within the time period of five years, a reduction in neonatal mortality rates is observed (Table 2).

For over a decade, the KHDSS has been a platform for a variety of studies. At the outset, various epidemiological studies were conducted in the area of infectious diseases of children, identifying signs and symptoms in young infant requiring urgent referral, vaccine coverage and the impact of multiple interventions. The focus was on measuring burden of relevant and common childhood illnesses. Some of these projects include: calculation of the incidence of various infectious diseases like typhoid bacteremia, pneumonia and diarrhea, evaluation of effectiveness of various treatment regimens for neonatal sepsis, assessment of the acceptance of hospitalized care, determining etiology of moderate to severe diarrhea, assessment of burden and etiology of neonatal sepsis and a multi-center cohort measuring the burden of stillbirths, neonatal and maternal deaths. (1-5)

\section{Conclusions}

All the studies aim for improvement of public health policies and informed decision making at local and national levels. We have also established a bio-repository of a well-defined maternal and newborn cohort.
Demographic Surveillance System Profile 2016

\begin{tabular}{|c|c|}
\hline Indicators & 2016 \\
\hline Total Population & 299,009 \\
\hline Total Area & $17.6 \mathrm{Sq} . \mathrm{Km}$ \\
\hline Total Structures & 42,093 \\
\hline Total Households & 43,098 \\
\hline Population Density/Sq. Km & 16,964 \\
\hline Total Male, $\mathbf{n}(\%)$ & $155,485(52)$ \\
\hline Total Female, $\mathbf{n}(\%)$ & $143,524(48)$ \\
\hline Total population 15-49years (females) & $74,752(25)$ \\
\hline Married women, $\mathbf{n}(\%)$ & $43,448(15)$ \\
\hline Children $<5$ years, $\mathbf{n}(\%)$ & $40,998(14)$ \\
\hline Annual pregnancies & 8264 \\
\hline Annual Livebirths & 7525 \\
\hline
\end{tabular}

Demographic Surveillance indicators and Trends by Year (2012-2016)

\begin{tabular}{|c|c|c|c|c|c|}
\hline Indicators (rates/ratios) & 2012 & 2013 & 2014 & 2015 & 2016 \\
\hline Crude birth rate(CBR)/1000 population & 29.9 & 27.3 & 26 & 28.7 & 25.2 \\
\hline Maternal mortality ratio(MMR)/100,000 Live births & 426.9 & 361 & 427 & 373.6 & 336.2 \\
\hline Neonatal mortality rate(NMR)/1000 live births & 44.8 & 51 & 42.3 & 37 & 39.5 \\
\hline Infant mortality rate(IMR)/1000 live births & 66.7 & 77 & 65.8 & 58 & 62.5 \\
\hline Under-five mortality rate(U5MR)/1000 live births & 78.5 & 89 & 77.2 & 70.9 & 76.7 \\
\hline Stillbirth rate(SBR)/1000 Births & 26.9 & 33 & 34.8 & 27 & 30.3 \\
\hline Pregnancy rate/1000 Women aged 15-49 Years & 186.6 & 163.2 & 157 & 183.4 & 185.6 \\
\hline Abortion rate/1000 Women aged 15-49 years & 5 & 5.8 & 4 & 6.1 & 7 \\
\hline General fertility rate (GFR)/1000 Women aged 15-49 & 119.6 & 109.2 & 104 & 114.9 & 100.7 \\
\hline Sex ratio (male to Female) & 1.1 & 1.1 & 1.1 & 1.1 & 1.1 \\
\hline Child-woman ratio & 707 & 683 & 629 & 608 & 548 \\
\hline In-migration/1000 Midyear Population & 54.3 & 44.2 & 33.7 & 21.1 & 25.0 \\
\hline Out-migration/1000 Midyear Population & 20 & 13.5 & 6.4 & 3.9 & 2.8 \\
\hline Crude net migration rate & 34.3 & 30.8 & 27.3 & 17.1 & 22.2 \\
\hline
\end{tabular}

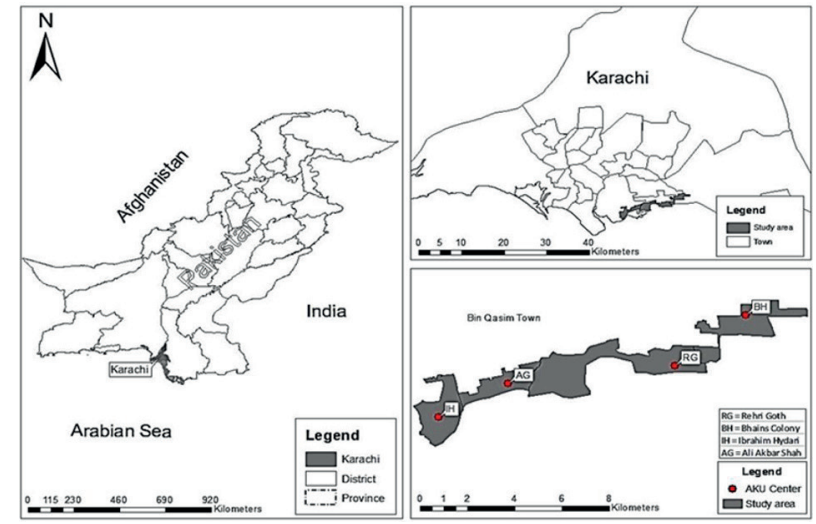

Figure 1: Map showing location of the DSS sites in Karachi, Pakistan.

\section{Keywords}

Karachi Health and Demographic Surveillance System; low socioeconomic communities; child and maternal health; research platform; Etiological studies and controlled trials

\section{References}

1. Group YICSS. Clinical signs that predict severe illness in children under age 2 months: a multicentre study. The Lancet. 2008; 371(9607):135-42. 
2. Kotloff KL, Nataro JP, Blackwelder WC, Nasrin D, Farag TH, Panchalingam S, et al. Burden and aetiology of diarrhoeal disease in infants and young children in developing countries (the Global Enteric Multicenter Study, GEMS): a prospective, case-control study. The Lancet. 2013;382(9888):209-22.

3. Mir F, Nisar I, Tikmani SS, Baloch B, Shakoor S, Jehan F, et al. Simplified antibiotic regimens for treatment of clinical severe infection in the outpatient setting when referral is not possible for young infants in Pakistan (Simplified Antibiotic Therapy Trial [SATT]): a randomised, open-label, equivalence trial. The Lancet Global Health. 2016.

4. Shafiq Y, Nisar MI, Kazi AM, Ali M, Jamal S, Ilyas M, et al. Implementation of the ANISA Study in Karachi, Pakistan: Challenges and Solutions. The Pediatric infectious disease journal. 2016;35(5):S60-S4.

5. group As. Burden, timing and causes of maternal and neonatal deaths and stillbirths in sub-Saharan Africa and South Asia: protocol for a prospective cohort study. Journal of Global Health. 2016;6(2).

\section{*Komal Naeem}

E-mail: komal.naeem@aku.edu 